International Journal of Pure and Applied Mathematics

Volume 84 No. 2 2013, 15-37

ISSN: 1311-8080 (printed version); ISSN: 1314-3395 (on-line version)

url: http://www.ijpam.eu

doi: http://dx.doi.org/10.12732/ijpam.v84i2.2

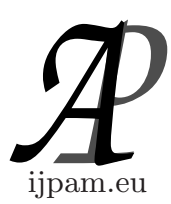

\title{
UNSTEADY FREE CONVECTION HEAT AND MASS TRANSFER IN AN MHD MICROPOLAR FLUID IN THE PRESENCE OF THERMO DIFFUSION AND THERMAL RADIATION
}

\author{
B.I. Olajuwon ${ }^{1}$, J.I. Oahimire ${ }^{2}$ \\ ${ }^{1}$ Department of Mathematics \\ Federal University of Agriculture \\ Abeokuta, NIGERIA \\ ${ }^{2}$ Department of Mathematics \\ University of Port Harcourt \\ Port Harcourt, NIGERIA
}

\begin{abstract}
This present study investigates the effects of thermo-diffusion and thermal radiation on unsteady heat and mass transfer of free convective MHD micropolar fluid flow bounded by a semi- infinite porous plate in a rotating frame under the action of transverse magnetic field with suction. The plate is assumed to oscillate in time with constant frequency so that the solutions of the boundary layer are the same oscillatory type. The governing system of partial differential equations is transformed to dimensionless equations using dimensionless variables. The dimensionless equations are then solved analytically using perturbation technique. With the help of graphs, the effects of the various important parameters entering into the problem on the velocity, microrotation, temperature and concentration fields within the boundary layer are discussed. Also the effects of the pertinent parameters on the local skin friction coefficient and rates of heat and mass transfer in terms of the local Nusselt and Sherwood numbers are presented numerically in tabular form. The results shows that
\end{abstract}

Received: June 6, 2012

(c) 2013 Academic Publications, Ltd. url: www.acadpubl.eu

$\S$ Correspondence author 
the observed parameters have significance influence on the flow, heat and mass transfer.

Key Words: micropolar fluid, dufour, perturbation technique, suction, heat and mass transfer, rotating frame

\section{Introduction}

Micropolar fluids are a subset of the micromorphic fluid theory introduced in a pioneering paper by Eringen[14]. Micropolar fluids are those consisting of randomly oriented particles suspended in a viscous medium, which can undergo a rotation that can affect the hydrodynamics of the flow, making it a distinctly non- Newtonian fluid. They constitute an important branch of non-Newtonian fluid dynamics where microrotation effects as well as microinertia are exhibited. Eringen's theory has provided a good model for studying a number of complicated fluids, such as colloidal fluids, polymeric fluids and blood.

The effects of radiation on unsteady free convection flow and heat transfer problem have become more important industrially. At high operating temperature, radiation effect can be quite significant. Many processes in engineering areas occur at high temperature and a knowledge of radiation heat transfer becomes very important for design of reliable equipments, nuclear plants, gas turbines and various propulsion devices or aircraft, missiles, satellites and space vehicles. Dufour effect referred to the heat flux produced by a concentration gradient. The effects are important for intermediate molecular weight gases in coupled heat and mass transfer in binary systems, often encountered in chemical process engineering and also in high-speed aerodynamics. Based on these applications, Cogley e t al.[13] showed that in the optically thin limit, the fluid does not absorb it's own emitted radiation but the fluid does absorb radiation emitted by the boundaries. Hossain and Takhar[17] have considered the radiation effects on mixed convection boundary layer flow of an optically dense viscous incompressible fluid along a vertical plate with uniform surface temperature. Makinde[18] examined the transient free convection interaction with thermal radiation of an absorbing emitting fluid along moving vertical permeable plate. Satter and Hamid[16] investigated the unsteady free convection interaction with thermal radiation of an absorbing emitting plate. Rahman and Satter[4] studied transient convective flow of micropolar fluid past a continuous moving porous plate in the presence of radiation. Heat and mass transfer effects on unsteady magneto hydrodynamics free convection flow near a moving vertical plate embedded in a porous medium was presented by Das and Jana[19]. 
Olajuwon[15] examine convection heat and mass transfer in a hydromagnetic flow of a second grade fluid past a semi-infinite stretching sheet in the presence of thermal radiation and thermo diffusion. Haque et al.[20] studied micropolar fluid behavior on steady magneto hydrodynamics free convection flow and mass transfer through a porous medium with heat and mass fluxes. Soret and dufour effects on mixed convection in a non- Darcy porous medium saturated with micropolar fluid was studied by Srinivascharya[12]. Rebhi[9] studied unsteady natural convection heat and mass transfer of micropolar fluid over a vertical surface with constant heat flux. The governing equations were solved numerically using McCormack's technique and effects of various parameters were investigated on the flow. Eldabe and Ouaf[6] solved the problem of heat and mass transfer in a hydromagnetic flow of a micropolar fluid past a stretching surface with ohmic heating and viscous dissipation using the Chebyshev finite difference method. Keelson and Desseaux [5] studied the effect of surface conditions on the flow of a micropolar fluid driven by a porous stretching surface. The governing equations were solved numerically. Sunil et al.[1] studied the effect of rotation on a layer of micropolar ferromagnetic fluid heated from below saturating a porous medium. The resulted non-linear coupled differential equations from the transformation were solved using finite-difference method. Mahmoud [7] investigated thermal radiation effect on magneto hydrodynamic flow of a micropolar fluid over a stretching surface with variable thermal conductivity. The solution was obtained numerically by iterative, Runge-Kuta order-four method. Magdy[10] studied unsteady free convection flow of an incompressible electrically conducting micropolar fluid, bounded by an infinite vertical plane surface of constant temperature with thermal relaxation including heat sources. The governing equations were solved using Laplace transformation. The inversion of the Laplace transforms was carried out with a numerical method. The obtained self-similar equation were solved numerically by an efficient implicit, iterative, infinite-difference method. Reena and Rana[8] investigated doublediffusive convection in a micropolar fluid layer heated and soluted from below saturating a porous medium. A linear stability analysis theory and normal mode analysis method was used. Kandasamy et al.[22] studied the nonlinear MHD flow, with heat and mass transfer characteristics, of an incompressible, viscous, electrically conducting, Boussinesq fluid on a vertical stretching surface with chemical reaction and thermal stratification effects. Modather et al.[21] studied MHD heat and mass transfer oscillatory flow of a micropolar fluid over a vertical permeable plate in a porous medium. Seddek[26] studied the effects of chemical reaction, thermophoresis and variable viscosity on steady hydromagnetic flow with heat and mass transfer over a flat plate in the presence of heat 
generation/absorption. Patil and Kulkarni[3] studied the effects of chemical reaction flow of a polar fluid through porous medium in the presence of internal heat generation. Double-diffusive convection radiation interaction on unsteady MHD flow over a vertical moving porous plate with heat generation and soret effects was studied by Mohamed[23]. Khedr et al.[25] studied MHD flow of a micropolar fluid past a stretched permeable surface with heat generation or absorption.

Unsteady free convection non-Newtonian fluids have many applications in geophysics, turbomachinery and many other fields. Motivated by these applications and previous work done, we studied the effects of thermo diffusion and thermal radiation on unsteady free convection heat and mass transfer flow of a micropolar fluid past a vertical porous plate in a rotating frame of reference with suction. It is assumed that the plate is embedded in a uniform porous medium and oscillate in time with a constant frequency . The governing equations are solved analytically using perturbation method. Different values of physical parameters are tabulated and discussed numerically and graphically

\section{Mathematical Formulation}

Consider three dimensional unsteady MHD flow of an incompressible, electrically conducting micropolar fluid past a semi-infinite vertical permeable moving plate embedded in a uniform porous medium in the presence of thermal radiation and thermo diffusion with suction. The flow is subjected to a constant transverse magnetic field $B_{0}$ in the presence of thermal and concentration buoyancy. The $\mathrm{x}$-direction is taken along the plate in the upward direction and z-axis is normal to it as shown in figure 1

The surface of the plate is held at a constant heat flux $q_{w}$, and the mass flux of a certain constituent in the solution that saturated the porous medium is held at $m_{w}$ near the surface. Due to the semi-infinite plane surface assumption, the flow variables are functions of $\mathrm{z}$ and $\mathrm{t}$ only. It is assumed that there is no applied voltage which implies the absence of an electric field. The flow is assumed to be in the x-direction which is taken along the plate. Also it is assumed that the whole system rotate with a constant frame $\Omega$ in a micropolar fluid about z-axis. The radiation heat flux in $\mathrm{x}$-direction is considered negligible in comparison with that in the z-direction.

Under these assumptions, the governing equation of the flow are:

$$
\frac{\partial w}{\partial z}=0
$$




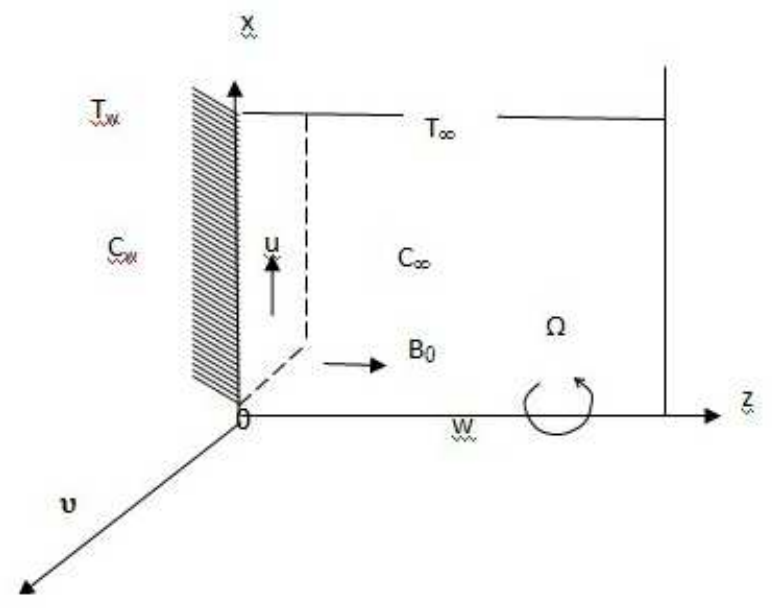

Figure 1: Physical model

$$
\begin{gathered}
\frac{\partial u}{\partial t}+w \frac{\partial u}{\partial z}-2 \Omega v \\
=\left(\nu+\nu_{r}\right) \frac{\partial^{2} u}{\partial z^{2}}+g \beta_{T}\left(T-T_{\infty}\right)+g \beta_{c}\left(C-C_{\infty}\right)-\frac{\nu u}{k}-\sigma \frac{B_{0}^{2} u}{\rho}-\nu_{r} \frac{\partial \bar{w}_{2}}{\partial z} \\
\frac{\partial v}{\partial t}+w \frac{\partial v}{\partial z}+2 \Omega u=\left(\nu+\nu_{r}\right) \frac{\partial^{2} v}{\partial z^{2}}-\frac{\nu v}{k}-\sigma \frac{B_{0}^{2} v}{\rho}+\nu_{r} \frac{\partial \bar{w}_{1}}{\partial z} \\
\frac{\partial \bar{w}_{1}}{\partial t}+w \frac{\partial \bar{w}_{1}}{\partial z}=\frac{\Lambda}{\rho j} \frac{\partial^{2} \bar{w}_{1}}{\partial z^{2}} \\
\frac{\partial \bar{w}_{2}}{\partial t}+w \frac{\partial \bar{w}_{2}}{\partial z}=\frac{\Lambda}{\rho j} \frac{\partial^{2} \bar{w}_{2}}{\partial z^{2}} \\
\frac{\partial T}{\partial t}+w \frac{\partial T}{\partial z}=\frac{\partial^{2} T}{\rho C_{p}} \frac{1}{\partial z^{2}}-\frac{\partial q_{r}}{\rho C_{p}}+\frac{D_{m} K_{T}}{\rho c} \frac{\partial^{2} C}{\partial z^{2}} \\
\frac{\partial C}{\partial t}+w \frac{\partial C}{\partial z}=D_{m} \frac{\partial^{2} C}{\partial z^{2}}
\end{gathered}
$$

Here $\mathrm{u}, v$ and $\mathrm{w}$ are velocity components along $\mathrm{x}, \mathrm{y}$, and z-axis respectively. $\bar{w}_{1}$ and $\bar{w}_{2}$ are microrotation components along $\mathrm{x}$ and $\mathrm{y}$-axis respectively. $\beta_{T}, \beta_{c}$ are the coefficient of thermal expansion and concentration expansion, $\rho$ is the density of the fluid, $\nu$ is the kinematic viscosity, $\nu_{r}$ is the kinematic microrotation viscosity, $\mathrm{k}$ is the permeability of porous medium, $\sigma$ is the electrical conductivity of the fluid, $\Lambda$ is the spin gradient velocity, $\mathrm{j}$ is the micro-inertia density, $\mathrm{g}$ 
is the acceleration due to gravity, $\mathrm{T}$ is the temperature of the fluid in the boundary layer while $T_{\infty}$ is the temperature far from the surface, $\kappa$ is the thermal conductivity of the mediun, $C_{p}$ is the specific heat at constant pressure $\mathrm{p}, q_{r}$ is the radiation heat flux, $\mathrm{C}$ is the concentration of the solute while $C_{\infty}$ is the concentration of the solute far from the surface, $D_{m}$ is the molecular diffusivity and $K_{T}$ is the thermo diffusion ratio and c is the concentration susceptibility. The boundary conditions are given by:

$$
\begin{gathered}
u=v=0, \bar{w}_{1}=\bar{w}_{2}=0, T=T_{\infty}, C=C_{\infty}, \text { for } t \leq 0, \\
u=U_{r}\left[1+\frac{\epsilon}{2}\left(e^{i n t}+e^{-i n t}\right], \quad v=0, \quad \bar{w}_{1}=-\frac{i}{2} \frac{\partial v}{\partial z},\right. \\
\bar{w}_{2}=\frac{i}{2} \frac{\partial u}{\partial z}, \quad-\kappa \frac{\partial T}{\partial z}=q_{w}, \\
-D_{m} \frac{\partial C}{\partial z}=M_{w} \text { at } x=0 \text { and } u=v=0, \\
\bar{w}_{1}=\bar{w}_{2}=0, T=T_{\infty}, C=C_{\infty} \text { as } z \rightarrow \infty \text { for } t>0 .
\end{gathered}
$$

Here $U_{r}$ is the uniform reference velocity and $\epsilon$ is the smallest constant quantity. The oscillatory plate velocity assumed in equation (9) is based on the suggestion proposed by [Ganapathy[24]]. From equation (1), we have

$$
w=-w_{0}
$$

Here $w_{0}$ represents the normal velocity at the plate which is positive for suction and negative for blowing. Following Rosseland approximation (Brewstar[11]) the radiative heat flux $q_{r}$ is modeled as

$$
q_{r}=-\frac{4 \sigma^{*}}{3 k^{*}} \frac{\partial T^{4}}{\partial z} .
$$

Here $\sigma^{*}$ is the Stefan-Boltzman constant and $\mathrm{k}^{*}$ is the mean absorption coefficient. Assuming that the difference in temperature within the flow are such that $T^{4}$ can be expressed as a linear combination of the temperature, we expand $T^{4}$ in Taylor's series about $T_{\infty}$ as follows:

$$
T^{4}=T_{\infty}^{4}+4 T_{\infty}^{3}\left(T-T_{\infty}\right)+6 T_{\infty}^{2}\left(T-T_{\infty}\right)^{2}+\ldots \ldots
$$

and neglecting higher order terms beyond the first degree $\operatorname{in}\left(T-T_{\infty}\right)$, we have

$$
T^{4} \approx-3 T_{\infty}^{4}+4 T_{\infty}^{3} T,
$$


Differentiating equation (11) with respect to $\mathrm{z}$ and using equation(13) to obtain

$$
\frac{\partial q_{r}}{\partial z}=-\frac{16 T_{\infty}^{3} \sigma^{*}}{3 k^{*}} \frac{\partial^{2} T}{\partial z^{2}}
$$

Let us introduce the following dimensionless variables:

$$
\begin{gathered}
u^{\prime}=\frac{u}{U_{r}}, v^{\prime}=\frac{v}{U_{r}}, z^{\prime}=\frac{z U_{r}}{\nu}, t^{\prime}=\frac{t U_{r}^{2}}{\nu}, n^{\prime}=\frac{n \nu}{U_{r}^{2}}, \bar{w}_{1}^{\prime}=\frac{\bar{w}_{1} \nu}{U_{r}^{2}}, \bar{w}_{2}^{\prime}=\frac{\bar{w}_{2} \nu}{U_{r}^{2}} \\
\theta=\frac{\kappa\left(T-T_{\infty}\right)}{q_{w}}, \phi=\frac{D_{m}\left(C-C_{\infty}\right)}{M_{w}}
\end{gathered}
$$

Substituting equation (15) into equations (2) - (7) and dropping primes yield the following dimensionless equations:

$$
\begin{gathered}
\frac{\partial u}{\partial t}-S \frac{\partial u}{\partial z}-R v=(1+\lambda) \frac{\partial^{2} u}{\partial z^{2}}+G_{r} \theta+G_{m} \phi-\left(M^{2}+\frac{1}{\chi}\right) u-\lambda \frac{\partial \bar{w}_{2}}{\partial z} \\
\frac{\partial v}{\partial t}-S \frac{\partial v}{\partial z}+R u=(1+\lambda) \frac{\partial^{2} v}{\partial z^{2}}-\left(M^{2}+\frac{1}{\chi}\right) v+\lambda \frac{\partial \bar{w}_{1}}{\partial z} \\
\frac{\partial \bar{w}_{1}}{\partial t}-S \frac{\partial \bar{w}_{1}}{\partial z}=K \frac{\partial^{2} \bar{w}_{1}}{\partial z^{2}} \\
\frac{\partial \bar{w}_{2}}{\partial t}-S \frac{\partial \bar{w}_{2}}{\partial z}=K \frac{\partial^{2} \bar{w}_{2}}{\partial z^{2}} . \\
\frac{\partial \theta}{\partial t}-S \frac{\partial \theta}{\partial z}=\frac{1}{P r}\left(1+\frac{4 N r}{3}\right) \frac{\partial^{2} \theta}{\partial z^{2}}+D_{f} \frac{\partial^{2} \phi}{\partial z^{2}} \\
\frac{\partial \phi}{\partial t}-S \frac{\partial \phi}{\partial z}=\frac{1}{S c} \frac{\partial^{2} \phi}{\partial z^{2}}
\end{gathered}
$$

Here $R=\frac{2 \Omega \nu}{U_{r}^{2}}$ is the rotational parameter, $M=\frac{B_{0}}{U_{r}} \sqrt{\frac{\sigma \nu}{\rho}}$ is the magnetic field parameter, $\operatorname{Pr}=\frac{\mu \rho C_{p}}{\kappa}, S c=\frac{\nu}{D_{m}}, G_{r}=\frac{\nu g \beta_{T} q_{w}}{\kappa U_{r}^{3}}, G_{m}=\frac{\nu g \beta_{c} M_{w}}{D_{m} U_{r}^{3}}$ are Prandtl number, Schmidt number, Grashof and modified Grashof number respectively. $N r=\frac{4 T_{\infty}^{3} \sigma^{*}}{\kappa K^{*}}$ is the heat radiation parameter, $\chi=\frac{k U_{r}^{2}}{\nu^{2}}$ is the permeability of the porous medium parameter, $D_{f}=\frac{\kappa^{2} K_{T} M_{w}}{q_{w} \rho c \mu}$ is the Dufour number, $S=\frac{w_{0}}{U_{r}}$ is the suction parameter, $K=\frac{\Lambda}{\mu j}$ is the dimensionless material parameter, $\lambda=\frac{\nu_{r}}{\nu}$ is the viscosity ratio.

Also the boundary conditions become

$$
u=v=0, \bar{\omega}_{1}=\bar{\omega}_{2}=0, \theta=0, \phi=0, \text { fort } \leq 0
$$




$$
\begin{gathered}
u=1+\frac{\epsilon}{2}\left(e^{i n t}+e^{-i n t}\right], v=0, \bar{w}_{1}=-\frac{i}{2} \frac{\partial v}{\partial z}, \bar{w}_{2}=\frac{i}{2} \frac{\partial u}{\partial z}, \theta^{\prime}=-1, \\
\phi^{\prime}=-1 \text { at } z=0, \text { and } u=v=0, \bar{\omega}_{1}=\bar{\omega}_{2}=0, \theta=\phi=0 \text { as } z \rightarrow \infty, \text { for } t>0
\end{gathered}
$$

We simplify equation(16) - (21) further by putting the fluid velocity and angular velocity in the complex form as $V=u+i v, w=\bar{w}_{1}+i \bar{w}_{2}$ and have the following

$$
\begin{gathered}
\frac{\partial V}{\partial t}-S \frac{\partial V}{\partial z}+i R v=(1+\lambda) \frac{\partial^{2} V}{\partial z^{2}}-\left(M^{2}+\frac{1}{\chi}\right) V+G_{r} \theta+G_{m} \phi+i \lambda \frac{\partial \bar{w}_{2}}{\partial z} \\
\frac{\partial w}{\partial t}-S \frac{\partial w}{\partial z}=K \frac{\partial^{2} w}{\partial z^{2}} \\
\frac{\partial \theta}{\partial t}-S \frac{\partial \theta}{\partial z}=\frac{1}{P r}\left(1+\frac{4 N r}{3}\right) \frac{\partial^{2} \theta}{\partial z^{2}}+D_{f} \frac{\partial^{2} \phi}{\partial z^{2}} \\
\frac{\partial \phi}{\partial t}-S \frac{\partial \phi}{\partial z}=\frac{1}{S c} \frac{\partial^{2} \phi}{\partial z^{2}}
\end{gathered}
$$

The associated boundary conditions (22) and (23) becomes

$$
\begin{gathered}
V=0, w=0, \theta=0, \phi=0, \text { fort } \leq 0 \\
V=1+\frac{\epsilon}{2}\left(e^{i n t}+e^{-i n t}\right], w=\frac{i}{2} \frac{\partial V}{\partial z}, \theta^{\prime}=-1, \\
\phi^{\prime}=-1 \text { at } z=0, \\
\text { and } V=0, w=0, \theta=\phi=0 \text { as } z \rightarrow \infty, \text { for } t>0
\end{gathered}
$$

\section{Method of Solution}

To find the solution of the above system of partial differential equations equations $(24)-(27)$ in the neighborhood of the plate under the above boundary conditions (28), (29), we assume a perturbation of the form:

$$
\begin{aligned}
V(z, t) & =V_{0}(z)+\frac{\epsilon}{2}\left[e^{i n t} V_{1}(z)+e^{-i n t} V_{2}(z)\right] \\
w(z, t) & =w_{0}(z)+\frac{\epsilon}{2}\left[e^{i n t} w_{1}(z)+e^{-i n t} w_{2}(z)\right] \\
\theta(z, t) & =\theta_{0}(z)+\frac{\epsilon}{2}\left[e^{i n t} \theta_{1}(z)+e^{-i n t} \theta_{2}(z)\right]
\end{aligned}
$$




$$
\phi(z, t)=\phi_{0}(z)+\frac{\epsilon}{2}\left[e^{i n t} \phi_{1}(z)+e^{-i n t} \phi_{2}(z)\right] .
$$

From equations (30) - (33), we have

$$
\begin{aligned}
& \frac{\partial V}{\partial z}=V_{0}^{\prime}+\frac{\epsilon}{2} e^{i n t} V_{1}^{\prime}+\frac{\epsilon}{2} e^{-i n t} V_{2}^{\prime} \\
& \frac{\partial w}{\partial z}=w_{0}^{\prime}+\frac{\epsilon}{2} e^{i n t} w_{1}^{\prime}+\frac{\epsilon}{2} e^{-i n t} w_{2}^{\prime} \\
& \frac{\partial \theta}{\partial z}=\theta_{0}^{\prime}+\frac{\epsilon}{2} e^{i n t} \theta_{1}^{\prime}+\frac{\epsilon}{2} e^{-i n t} \theta_{2}^{\prime} \\
& \frac{\partial \phi}{\partial z}=\phi_{0}^{\prime}+\frac{\epsilon}{2} e^{i n t} \phi_{1}^{\prime}+\frac{\epsilon}{2} e^{-i n t} \phi_{2}^{\prime} \\
& \frac{\partial^{2} V}{\partial z^{2}}=V_{0}^{\prime \prime}+\frac{\epsilon}{2} e^{i n t} V_{1}^{\prime \prime}+\frac{\epsilon}{2} e^{-i n t} V_{2}^{\prime \prime} \\
& \frac{\partial^{2} w}{\partial z^{2}}=w_{0}^{\prime \prime}+\frac{\epsilon}{2} e^{i n t} w_{1}^{\prime \prime}+\frac{\epsilon}{2} e^{-i n t} w_{2}^{\prime \prime} \\
& \frac{\partial^{2} \theta}{\partial z^{2}}=\theta_{0}^{\prime \prime}+\frac{\epsilon}{2} e^{i n t} \theta_{1}^{\prime \prime}+\frac{\epsilon}{2} e^{-i n t} \theta_{2}^{\prime \prime} \\
& \frac{\partial^{2} \phi}{\partial z^{2}}=\phi_{0}^{\prime \prime}+\frac{\epsilon}{2} e^{i n t} \phi_{1}^{\prime \prime}+\frac{\epsilon}{2} e^{-i n t} \phi_{2}^{\prime \prime} \\
& \frac{\partial V}{\partial t}=\frac{i n t}{2} e^{i n t} V_{1}-\frac{i n t}{2} e^{-i n t} V_{2} \\
& \frac{\partial w}{\partial t}=\frac{i n t}{2} e^{i n t} w_{1}-\frac{i n t}{2} e^{-i n t} w_{2} \\
& \frac{\partial \theta}{\partial t}=\frac{i n t}{2} e^{i n t} \theta_{1}-\frac{i n t}{2} e^{-i n t} \theta_{2} \\
& e^{i n t} \phi_{1}-\frac{i n t}{2} e^{-i n t} \phi_{2} \\
& \\
& \frac{\partial n t}{2}{ }^{i n t}
\end{aligned}
$$

Substitute equations (30) - (45) into equations (24) - (29), equating the harmonic and non-harmonic terms and neglecting the higher order terms of $O\left(\epsilon^{2}\right)$ to obtain the following set of equations:

$$
\begin{gathered}
(1+\lambda) V_{0}^{\prime \prime}+S V_{0}^{\prime}-C_{1} V_{0}+G_{r} \theta_{0}+G_{m} \phi_{0}+i \lambda w_{0}^{\prime}=0 \\
K w_{0}^{\prime \prime}+S w_{0}^{\prime}=0 \\
(3+4 N r) \theta_{0}^{\prime \prime}+3 S \operatorname{Pr} \theta_{0}^{\prime}+3 \operatorname{Pr} D_{f} \phi_{0}^{\prime \prime}=0 \\
\phi_{0}^{\prime \prime}+S S c \phi_{0}^{\prime}=0
\end{gathered}
$$




$$
\begin{gathered}
(1+\lambda) V_{1}^{\prime \prime}+S V_{1}^{\prime}-C_{2} V_{1}+G_{r} \theta_{1}+G_{m} \phi_{1}+i \lambda w_{1}^{\prime}=0, \\
K w_{1}^{\prime \prime}+S w_{1}^{\prime}-i n w_{1}=0 \\
(3+4 N r) \theta_{1}^{\prime \prime}+3 S \operatorname{Pr} \theta_{1}^{\prime}-3 \operatorname{Prin} \theta_{1}+3 \operatorname{Pr} D_{f} \phi_{1}^{\prime \prime}=0 \\
\phi_{1}^{\prime \prime}+S S c \phi_{1}^{\prime}-S \operatorname{cin} \phi_{1}=0, \\
(1+\lambda) V_{2}^{\prime \prime}+S V_{2}^{\prime}-C_{3} V_{2}+G_{r} \theta_{2}+G_{m} \phi_{2}+i \lambda w_{2}^{\prime}=0 \\
K w_{2}^{\prime \prime}+S w_{2}^{\prime}+i n w_{2}=0, \\
(3+4 N r) \theta_{2}^{\prime \prime}+3 S \operatorname{Pr} \theta_{2}^{\prime}+3 \operatorname{Prin} \theta_{2}+3 \operatorname{Pr} D_{f} \phi_{2}^{\prime \prime}=0, \\
\phi_{2}^{\prime \prime}+S S c \phi_{2}^{\prime}+\operatorname{Scin} \phi_{2}=0,
\end{gathered}
$$

Here the prime denote differentiation with respect to z and $C_{1}=i R+M^{2}+\frac{1}{\chi}$ ,$C_{2}=i(R+n)+M^{2}+\frac{1}{\chi}$ and $C_{3}=i(R-n)+M^{2}+\frac{1}{\chi}$ The corresponding boundary conditions can be written as

$$
\begin{gathered}
V_{0}=V_{1}=V_{2}=1, w_{0}=\frac{i}{2} V_{0}^{\prime}, w_{1}=\frac{i}{2} V_{1}^{\prime}, w_{2}=\frac{i}{2} V_{2}^{\prime}, \\
\theta_{0}^{\prime}=-1, \phi_{0}^{\prime}=-1, z=0, \\
V_{0}=V_{1}=V_{2}=0, w_{0}=w_{1}=w_{2}=0, \theta_{0}=\theta_{1}=\theta_{2}=0, \\
\phi_{0}=\phi_{1}=\phi_{2}=0, z \rightarrow \infty
\end{gathered}
$$

Solving equations (46) - (57) with equation (58) and (59) yield the expression for velocity, microrotation, temperature and concentration as follows:

$$
\begin{gathered}
V=A_{1} e^{-m_{3} z}+A_{2} e^{-m_{2} z}+A_{3} e^{-m_{1} z}+A_{4} e^{\frac{S}{K} z}+\frac{\epsilon}{2}\left[\left(A_{5} e^{-m_{5} z}+A_{6} e^{-m_{4} z}\right) e^{i n t}\right. \\
\left.+\left(A_{7} e^{-m_{7} z}+A_{8} e^{-m_{6} z}\right) e^{-i n t}\right] \\
w=B_{1} e^{-\frac{S}{K} z}+\frac{\epsilon}{2}\left[B_{2} e^{-m_{4} z} e^{i n t}+B_{3} e^{-m_{6} z} e^{-i n t}\right] \\
\theta=D_{1} e^{-m_{2} z}+D_{2} e^{-m_{1} z} \\
\phi=\frac{1}{m_{1}} e^{-m_{1} z}
\end{gathered}
$$

Here:

$$
\begin{aligned}
& m_{1}=S S c \\
& m_{2}=\frac{3 S P r}{3+4 N r} \\
& m_{3}=\frac{S+\sqrt{S^{2}+4(1+\lambda) C_{1}}}{2(1+\lambda)}
\end{aligned}
$$




$$
\begin{aligned}
& m_{4}=\frac{S+\sqrt{S^{2}+4 K i n}}{2 \lambda} \\
& m_{5}=\frac{S+\sqrt{S^{2}+4(1+\lambda) C_{2}}}{2(1+\lambda)} \\
& m_{6}=\frac{S+\sqrt{S^{2}-4 K i n}}{2 K} \\
& m_{7}=\frac{S+\sqrt{S^{2}+4(1+\lambda) C_{3}}}{2(1+\lambda)} \\
& D_{2}=\frac{-3 P r D_{f} m_{1}(3+4 N r)}{(3+4 N r) m_{1}^{2}-3 P r S m_{1}} \\
& D_{1}=\frac{1-m_{1} D_{2}}{m_{2}} \\
& A_{2}=\frac{-G_{r} D_{1}}{m_{2}^{2}(1+\lambda)-m_{2} S-C_{1}} \\
& A_{3}=\frac{-\left(G_{r} m_{1} D_{2}+G_{m}\right)}{m_{1}\left(m_{1}^{2}(1+\lambda)-m_{1} S-C_{1}\right)} \\
& A_{4}=\frac{K \lambda S\left(m_{3}-m_{3} A_{2}-m_{3} A_{3}+m_{2} A_{2}+m_{1} A_{3}\right)}{2\left(S^{2}(1+\lambda)-K S^{2}-K^{2} C_{1}\right)-\lambda S^{2}+K \lambda S m_{3}} \\
& A_{1}=1-A_{2}-A_{3}-A_{4} \\
& A_{6}=\frac{\lambda m_{4} m_{5}}{2\left((1+\lambda) m_{4}^{2}-S m_{4}-C_{2}\right)-\lambda m_{4}^{2}+\lambda m_{4} m_{5}} \\
& A_{5}=1-A_{6} \\
& A_{8}=\frac{\lambda m_{6} m_{7}}{2\left((1+\lambda) m_{6}^{2}-S m_{6}-C_{3}\right)-\lambda m_{6}^{2}+\lambda m_{6} m_{7}} \\
& A_{7}=1-A_{8} \\
& B_{1}=-\frac{i}{2}\left(m_{3} A_{1}+m_{2} A_{2}+m_{1} A_{3}+\frac{S}{K} A_{4}\right) \\
& B_{2}=-\frac{i}{2}\left(m_{5} A_{5}+m_{4} A_{6}\right) \\
& B_{3}=-\frac{i}{2}\left(m_{7} A_{7}+m_{6} A_{8}\right) \\
& \left.A_{1}\right)
\end{aligned}
$$

The results are presented as velocity, temperature and concentration profiles in figures 2 - 12 below;

For practical engineering applications, quantities of interest include skinfriction coefficient, couple stress coefficient, nusselt number and Sherwood number.

The local skin-friction coefficient is given by

$$
\begin{aligned}
& C_{f}=\frac{\tau_{w} \mid z=0}{\rho U_{r}^{2}}=\left[1+\lambda\left(1+\frac{i}{2}\right)\right] \frac{\partial V}{\partial z}=-\left[1+\lambda\left(1+\frac{i}{2}\right)\right]\left[m_{3} A_{1}+\right. \\
& m_{2} A_{2}+m_{1} A_{3}+\frac{S}{K} A_{4}+\frac{\epsilon}{2}\left[\left(m_{5} A_{5}+m_{4} A_{6}\right) e^{i n t}+\left(m_{7} A_{7}+m_{6} A_{8}\right) e^{-i n \text { (6) }])}\right]
\end{aligned}
$$

The couple stress coefficient at the wall $C_{w}$ is given by

$$
C_{w}=\left.\frac{\partial w}{\partial z}\right|_{z=0}=-\left[\frac{S}{K} B_{1}+\frac{\epsilon}{2}\left(m_{4} B_{2} e^{i n t}+m_{6} B_{3} e^{-i n t}\right)\right]
$$

The local Nusselt number $\mathrm{Nu}$ is given by

$$
N u=-\frac{x\left(\frac{\partial T}{\partial z}\right)_{z=0}}{K\left(T_{w}-T_{\infty}\right)}=\frac{R e_{x}}{\theta(0)}
$$




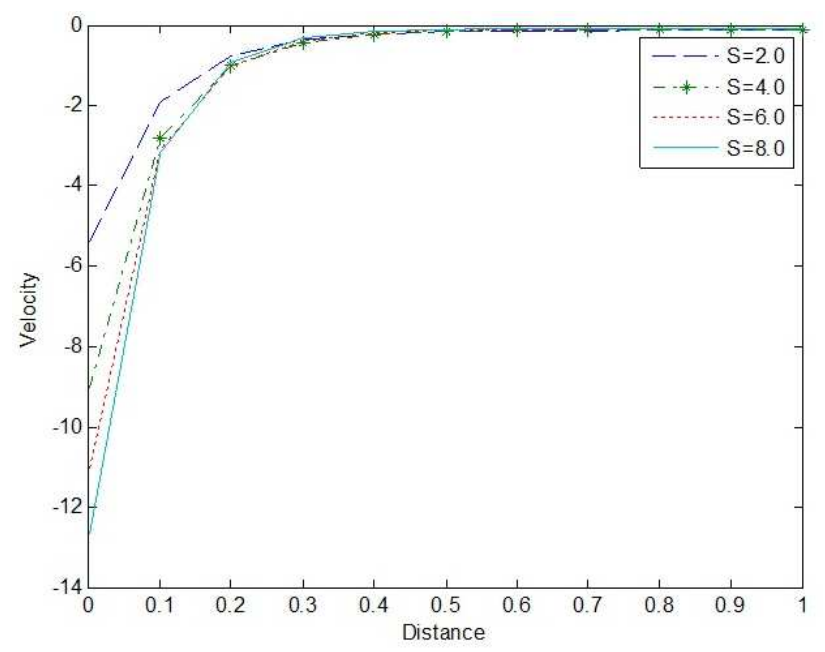

Figure 2: Velocity profiles for different values of suction parameter .

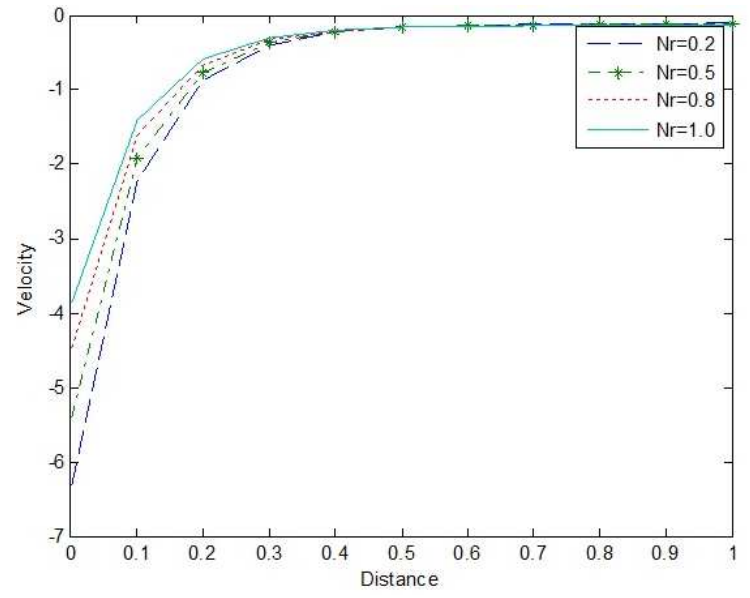

Figure 3: Velocity profiles for different values of radiation parameter .

Here $R e_{x}=\frac{U_{r} x}{\nu}$ is the local Reynolds number.

Thus

$$
\frac{N u}{R e_{x}}=\frac{1}{\theta(0)}
$$

Also the local Sherwood number Sh is given by $S h=-\frac{x\left(\frac{\partial C}{\partial z}\right)_{z=0}}{D_{m}\left(C_{w}-C_{\infty}\right)}=\frac{R e_{x}}{\phi(0)}$ 


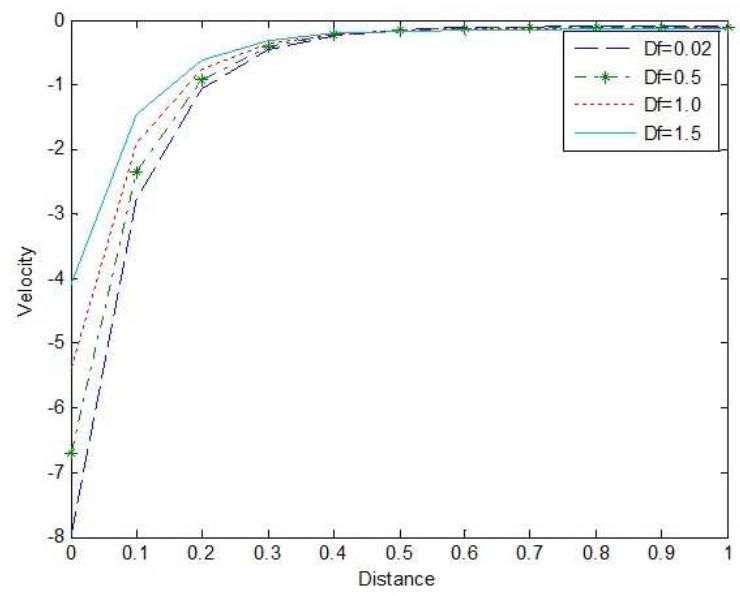

Figure 4: Velocity profiles for different values of thermo-diffusion parameter .

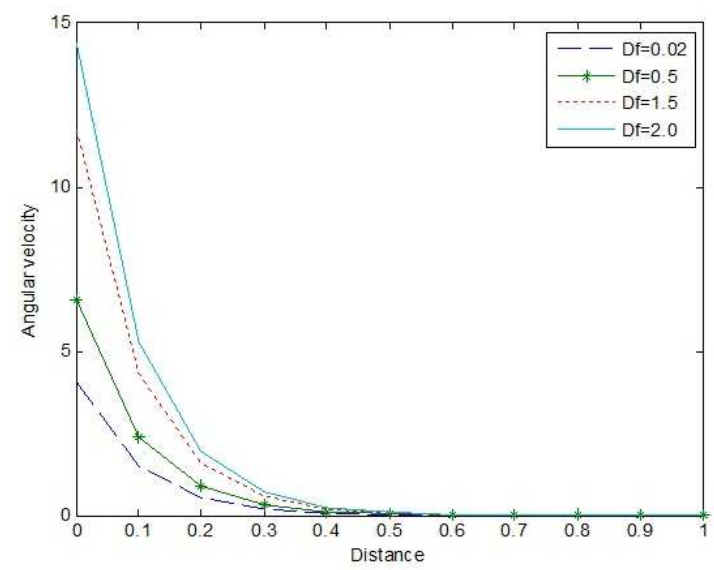

Figure 5: Microrotation profiles for different values of thermo-diffusion parameter .

Thus

$$
\frac{S h}{R e_{x}}=\frac{1}{\phi(0)}
$$

The numerical result for skin friction, Nusselt number and Sherwood number are shown in tables 1 - 4 below: 




Figure 6: Microrotation profiles for different values of radiation parameter.

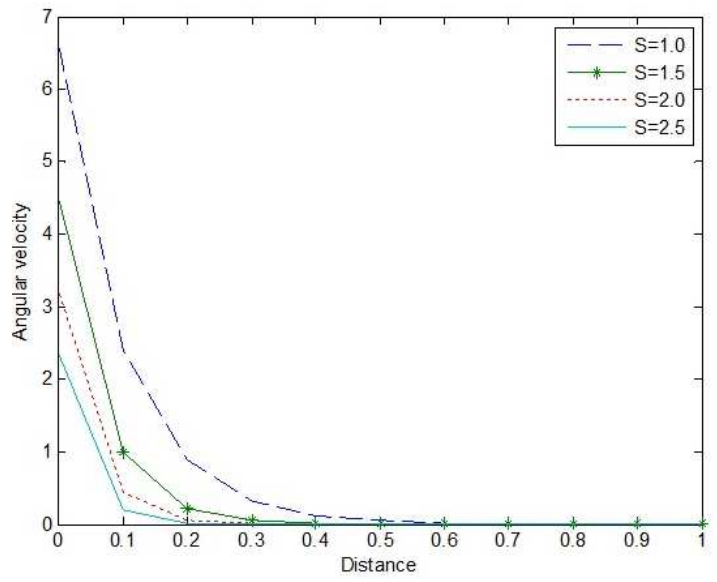

Figure 7: Microrotation profiles for different values of Suction parameter. 


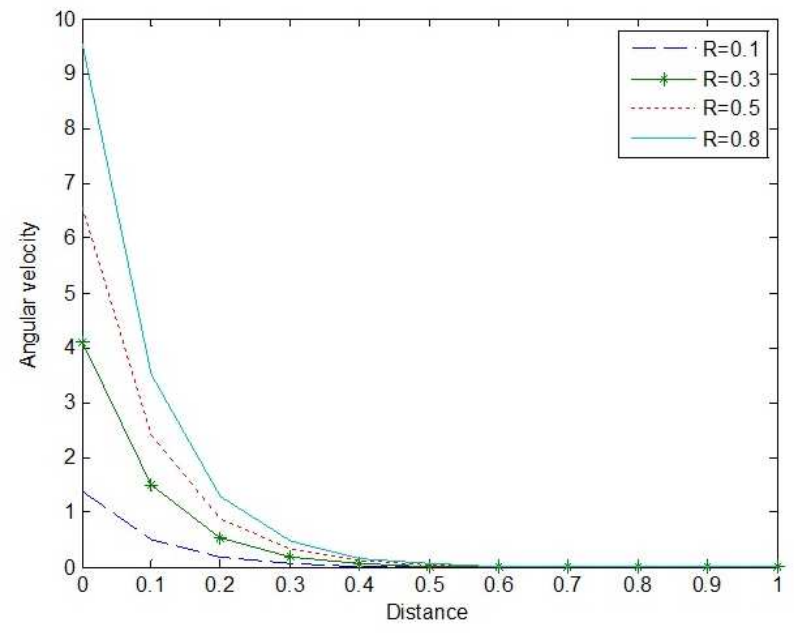

Figure 8: Microrotation profiles for different values of rotational parameter

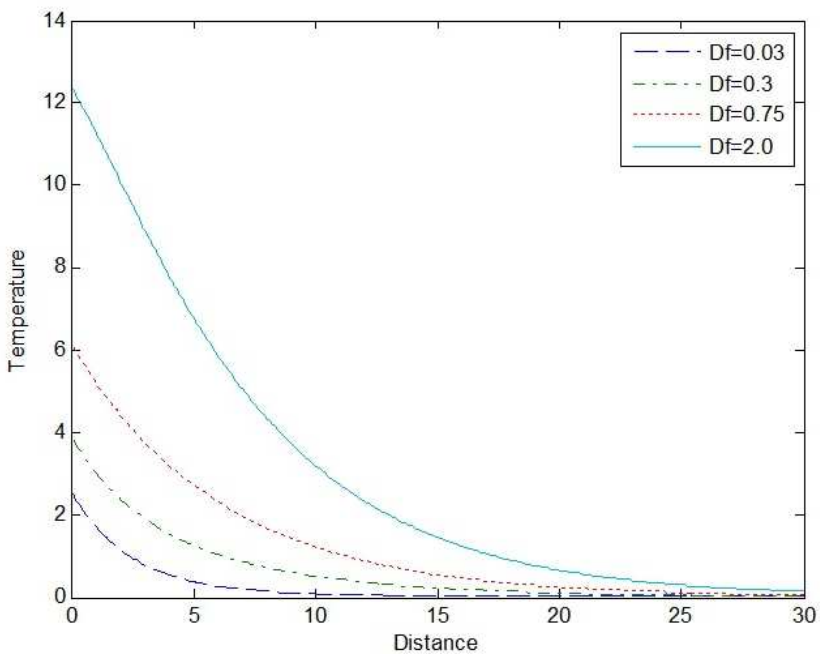

Figure 9: Temperature profiles for different values of Dufour parameter 


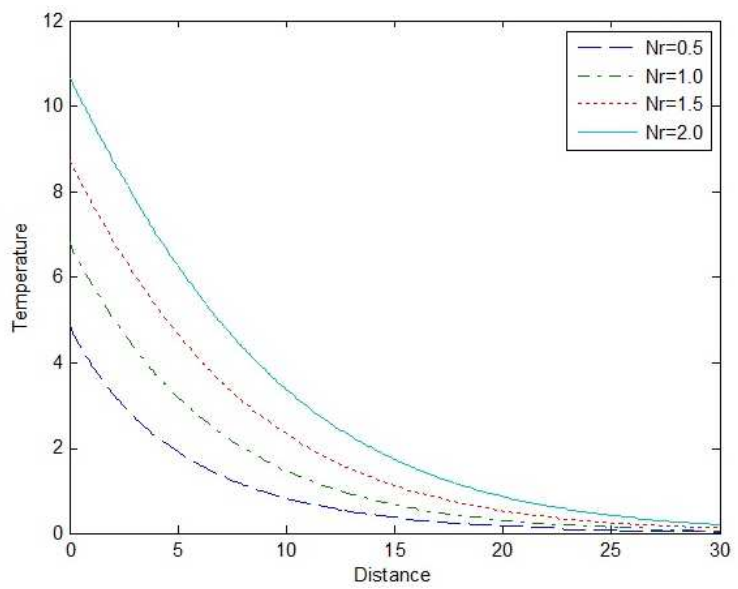

Figure 10: Temperature profiles for different values of radiation parameter.

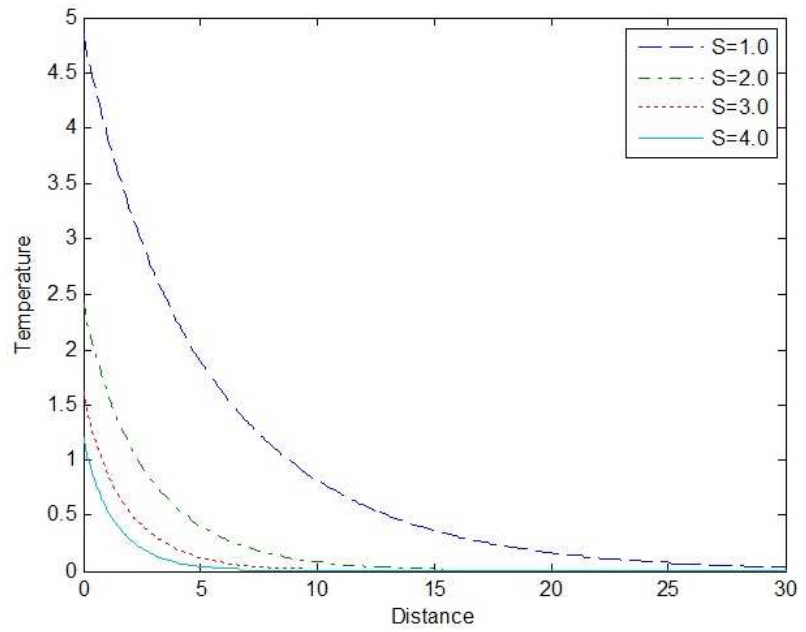

Figure 11: Temperature profiles for different values of Suction parameter. 




Figure 12: Concentration profiles for different values of suction parameter.

\section{Table1}

Effect of various parameter on $C_{f}$ with $\mathrm{K}=0.5, \mathrm{Sc}=0.16, \mathrm{Pr}=0.71, \mathrm{M}=10, \lambda=$ 0.1

\begin{tabular}{|c|c|c|c|c|}
$\mathrm{Nr}$ & $\mathrm{S}$ & $D_{f}$ & $\mathrm{R}$ & $C_{f}$ \\
\hline 0.5 & 2.5 & 0.02 & 0.5 & -10.5322 \\
1.0 & 2.5 & 0.02 & 0.5 & -10.0297 \\
0.5 & 2.5 & 0.02 & 0.5 & -9.5252 \\
0.5 & 4.0 & 0.02 & 0.5 & -11.8486 \\
0.5 & 5.0 & 0.02 & 0.5 & -12.9772 \\
0.5 & 6.0 & 0.02 & 0.5 & -13.9761 \\
0.5 & 2.5 & 0.3 & 0.5 & -8.2235 \\
0.5 & 2.5 & 0.5 & 0.5 & -7.2937 \\
0.5 & 2.5 & 1.0 & 0.5 & -4.9692 \\
0.5 & 2.5 & 0.02 & 0.8 & -9.5216 \\
0.5 & 2.5 & 0.02 & 1.0 & -9.5204 \\
0.5 & 2.5 & 0.02 & 1.5 & -9.5176 \\
\hline
\end{tabular}




\section{Table2}

Effect of various parameter on $-C_{w}$ with $\mathrm{K}=0.5, \mathrm{Sc}=0.16, \operatorname{Pr}=0.71, \mathrm{M}=10, \lambda=$ 0.1

\begin{tabular}{|c|c|c|c|c|}
$\mathrm{Nr}$ & $\mathrm{S}$ & $D_{f}$ & $\mathrm{R}$ & $-C_{w}$ \\
\hline 0.5 & 2.5 & 0.02 & 0.5 & 3.6452 \\
0.8 & 2.5 & 0.02 & 0.5 & 3.7305 \\
1.0 & 2.5 & 0.02 & 0.5 & 3.7879 \\
0.5 & 3.0 & 0.02 & 0.5 & 4.2471 \\
0.5 & 5.0 & 0.02 & 0.5 & 6.5960 \\
0.5 & 7.0 & 0.02 & 0.5 & 8.8088 \\
0.5 & 2.5 & 0.03 & 0.5 & 3.6526 \\
0.5 & 2.5 & 0.05 & 0.5 & 3.6673 \\
0.5 & 2.5 & 1.0 & 0.5 & 3.7042 \\
0.5 & 2.5 & 0.02 & 0.8 & 5.8323 \\
0.5 & 2.5 & 0.02 & 1.0 & 7.2903 \\
0.5 & 2.5 & 0.02 & 1.2 & 8.7483 \\
\hline
\end{tabular}

Table3

Effect of $\mathrm{Nr}, \mathrm{S}$ and $D_{f}$ on $N u R e_{x}^{-1}$ with $\mathrm{K}=0.5,=0, \mathrm{Sc}=0.16, \mathrm{Pr}=0.71, \mathrm{M}=10$, $?=0.1, \lambda=0.1$

\begin{tabular}{|c|c|c|c|}
$\mathrm{Nr}$ & $\mathrm{S}$ & $D_{f}$ & $\mathrm{Nu} \mathrm{Re}_{x}^{-1}$ \\
\hline 0.5 & 2.5 & 0.02 & 1.0215 \\
0.8 & 2.5 & 0.02 & 0.8238 \\
1.2 & 2.5 & 0.02 & 0.6548 \\
0.5 & 4.0 & 0.02 & 1.6344 \\
0.5 & 5.0 & 0.02 & 2.0430 \\
0.5 & 6.0 & 0.02 & 2.4516 \\
0.5 & 2.5 & 0.03 & 1.0010 \\
0.5 & 2.5 & 0.5 & 0.5157 \\
0.5 & 2.5 & 1.0 & 0.3403 \\
\hline
\end{tabular}




\section{Table4}

Effect of $\mathrm{S}$ on $S h R e_{x}^{-1}$ with $\mathrm{Sc}=0.16, \mathrm{~K}=0.5, \mathrm{Pr}=0.71, \lambda=0.1$.

\begin{tabular}{|c|c|}
$\mathrm{S}$ & $\mathrm{Sh} \mathrm{Re}_{x}^{-1}$ \\
\hline 4.0 & 0.6400 \\
5.0 & 0.8000 \\
6.0 & 0.9600 \\
\hline
\end{tabular}

\section{Discusson}

We have formulated the effect of thermo diffusion and radiation with suction on MHD free convection heat and mass transfer flow of an incompressible micropolar fluid along a semi-infinite vertical permeable moving plate embedded in a porous medium in a rotating frame of reference. The numerical calculation for distribution of the translational velocity, microrotation, temperature and concentration across the boundary layer for different values of of the parameters are carried out. For the purpose of our computation, we have chosen $n t=\frac{\pi}{2}$, $\mathrm{Pr}=0.71, \mathrm{Sc}=0.16, G_{m}=5, G_{r}=10, \mathrm{M}=10, \epsilon=0.01$ while $\mathrm{S}, \mathrm{Nr}, D_{f}$ and $\mathrm{R}$ are varied over range as shown in the figures.

Fig.2 shows the behavior of velocity profile with different values of suction parameter S. It was observed that velocity profiles decreases within the boundary layer with increase in suction. Thus the effects of increasing values of the suction parameter $\mathrm{S}$ is to decrease the momentum boundary layer thickness. Fig.3 shows the translational velocity across the boundary layer for different values of radiation parameter $\mathrm{Nr}$. It is depicted that velocity increase with the increase in the radiation parameter and as a result, the momentum boundary layer thickness increases. Fig.4 indicates the variation of velocity profiles with different values of thermo-diffusion parameter(or Dufour number) $D_{f}$. The fluid velocity increases with increase in Dufour number. The effect of increasing the value of the Dufour number is to increase the boundary layer. Fig5. Shows the influence of Dufour number in microrotation profiles. The microrotation profiles increases as the Dufour number increases. Fig.6 shows microrotation distribution across the boundary layer for different values of the heat radiation parameter Nr. It is observed that microrotation profiles increases with the increase in the heat radiation parameter and as a result, the momentum boundary layer thickness increases. The influence of the suction parameter S on microrotation distribution across the boundary layer is shown in fig.7. The result indicates that with increase in the parameter $\mathrm{S}$, microrotstion profiles 
decreases within the boundary layer region. Thus the effect of increasing values of suction parameter is to decrease the momentum boundary layer thickness. The microrotation profiles against distance for different values of rotational parameter $\mathrm{R}$ is displayed in fig.8. It shows that an increase in $\mathrm{R}$ leads to increase in microrotation distribution and so increase the momentum boundary layer thickness. Fig.9. shows the influence of Dufour number on temperature profiles. The temperature increases with increase in Dufour number. Fig.10 displays the variation of temperature profiles with different values of radiation parameter Nr. The temperature profiles increases as the radiation parameter increases and so increase the thermal boundary layer thickness. Fig.11 illustrates the variation of the suction parameter $S$ on the temperature profiles. It is observed that the temperature profiles decreases as the suction parameter increases which indicates that the thickness of the boundary layer is reduced for higher value of suction parameter. Fig.12 shows the variation of concentration profiles with different values of suction parameter S.The concentration of the fluid decreases as the suction parameter increases. Table1 depicts the effect of Nr, S, $D_{f}$ and R on the skin friction coefficient $C_{f}$. It is observed that the local skin friction coefficient increases as radiation parameter $\mathrm{Nr}$, Dufour number $D_{f}$ and rotational parameter $\mathrm{R}$ increases while it decreases as suction parameter increases. Table2 shows the effect of Nr, S, Df and R on couple stress coefficient. It is observed that the couple stress coefficient increases as these parameters increases. Table3 indicate the effect of Nr, S and $D_{f}$ on nusselt number. It is found that the nusselt number decreases as the $\mathrm{Nr}$ and $D_{f}$ increases while it increases as S increases. Table4 analyzed the effect of S on Sherwood number and it is also found that Sherwood number increases as suction parameter increases. These result are in good agreement with Rawat[2] and RamReddy[16].

\section{Conclusion}

The problem of unsteady free convection MHD heat and mass transfer flow of an incompressible micropolar fluid past a semi-infinite vertical permeable moving plate embedded in a uniform porous medium with suction in the presence of thermo-diffusion and thermal radiation was studied. The resulting partial differential equations which describe the problem, are transformed to dimensionless equations using dimensioless variables. Then solve the equations analytically by using perturbation technique. The results are discussed through graphs and tables for different values of parameters entering into the problem. 
Following conclusions can be drawn from the results obtained:

* The translational velocity decreases with increase in the value of suction parameter while it increases with an increasing radiation parameter and Dufour number.

* The magnitude of microrotation decreases with an increasing of suction parameter. Hence the momentum boundary layer thickness is reduced but the reverse effect is seen with an increase in the value of $\mathrm{Df}, \mathrm{Nr}$, and $\mathrm{R}$.

* The temperature profile increases with an increasing value of Dufour number and radiation parameter whereas the effect is opposite for suction parameter. Thus the boundary layer thickness increases for higher values of the radiation parameter.

* The concentration of the fluid decreases as the suction parameter increases.

\section{References}

[1] Sunil, A. Sharma, A. Bharti, P.K. and Shandi, R.G. Effect of rotation on a layer of micropolar ferromagnetic fluid heated from below saturating a porous medium, International Journal of Engineering science, vol. 44 no. 11-12, pp. 683-698, 2006.

[2] Rawat, S. and Bhargava, R. Finite element study of natural convection heat and mass transfer in a micropolar fluids-saturated porous regime with Soret/Dufour effects Int.J. of Appl. Maths and mech. 5(2): 58-71, 2009

[3] Patil, P.M. and Kulkarni P.S. Effects of chemical reaction on free convective flow of a polar fluid through a porous medium in the presence of internal heat generation, Int.Therm. Sci. 4. Pp. 1043-1054,2008

[4] Rahman, M.A. and Sattar, M.A. Transient convective flow of micropolar fluid past a continuously moving vertical porous plate in the presence of radiation, International Journal of Applied Mechanics and Engineering, vol.12, no. 2 pp.497-513, 2007.

[5] Keelson, N.A., Desseaux, A. Effects of surface condition on flow of a micropolar fluid driven by a porous stretching sheet, Int. J. Eng. Sci, 39, pp. 1881-1897, 2001.

[6] Eldabe, N.T. and Ouat, M.E., Chebyshew finite difference method for heat and mass transfer in hydromagnetic flow of a micropolar fluid past 
a stretching surface with Ohmic heating and viscous dissipation, Appl. Math. Comput., 177, pp. 561-571, 2006.

[7] Mahmoud, M.A.A., Thermal radiation effects on MHD flow of a micropolar fluid over a stretching surface with variable thermal conductivity, Physical A. 375 , pp. $401-410,2007$..

[8] Reena- I. and Rana U.S., Linear stability of thermo solutal convection in a micropolar fluid saturating a porous medium, International Journal of Application and Applied mathematics, vol. 4, no. 1 pp.62-87 2009

[9] Rehbi, A.D., Tariq, A.A. Benbella, A.S. Mahoud, A.A. Unsteady natural convection heat transfer of micropolar fluid over a vertical surface with constant Heat flux, Turkish J. Eng. Env. Sci. Vol. 31, pp. 225-233 (2007).

[10] Magdy, A.C., Free convection flow of conducting micropolar fluid with thermal relaxation including heat sources, Journal of Applied Mathematics, Vol. 2, 70.4 pp.271-292,2005.

[11] Brewster, M. Q., Thermal radiation transfer properties, John Wiley and sons, 12:6-9, 1972.

[12] Srinivasacharya.D., Ramreddy. C.,Soret and Dufour effect on mixed convection in a non-Darcy porous medium saturated with micropolar fluid, Non-analysis modelling and control, vol.16,No.1, 100-115,2011.

[13] Cogley A.C., Vincent W.E., Gilles S.E.Differential approximation for radiation in a non-gray gas near equilibrium. AIAAJ. 6:551-553,1968.

[14] Erigen A.C, Theory of micropolar fluids J. math.mech. 16, pp.1-18

[15] Olajuwon. B.I. Convection heat and mass transfer in a hydromagnetic flow of a second grade fluid in the presence of thermal radiation and thermal diffusion. Int. commu. Heat and mass 38:377-382,2007.

[16] Satter M.D. A, Hamid M.D. K. Unsteady free convection interaction with thermal radiation in a boundary layer flow past a vertical porous plate. Jour. Math. Phys. Sci. 30:25-37, 1996

[17] Hossain MA, Takhar Hs. Radiation effect on mixed convection along a vertical plate with uniform surface temperature, Heat mass transfer 31:243248, 1996 
[18] Makinde OD Free convection flow with thermal radiation and mass transfer past a moving vertical porous plate. Int. comm. Heat mass transfer, 25:289295,2005

[19] Das K, Jana S.Heat and mass transfer effects on unsteady MHD free convection flow near a moving vertical plate in a porous medium. Bull. Soc. Banja luka 17:15-32,2010

[20] Haque Md Z, Alam Md M, Ferdows M, Postelnicu A. Micropolar fluid behaviors on steady MHD free convection flow and mass transfer with constant heat and mass fluxes, joule heating and viscous dissipation. J.King Saud Univ. Engg.Sci doi:10.1016/j.jksues.2011.02.003, 2011.

[21] Modather M, Rashad A.M, Chamkha A.J. Study of MHD heat and mass transfer oscillatory flow of a micropolar fluid over a vertical permeable plate in a porous medium. Turkis J. eng. Env. Sci. vol33, 245-257, 2009.

[22] Kandasamy R., Periasamy K. and Sivagnana K. Chemical reaction, heat and mass transfer on MHD flow over a vertical stretching surface with heat source and stratification effects. Int. J. of heat and mass transfer, 48, 4557, 2005.

[23] Mohamad. Double diffusive convection-radiation interaction on unsteady MHD flow over a vertical moving porous plate with heat generation and soret effect, Applied mathematical sciences 13, pp. 629-651, 2009

[24] Ganapathy R. A note on oscillatory coquette flow in a rotating system. ASME J. Appl. Mech. 61; 208-209, 1994.

[25] Khedr M. E. , Chamkha A. J. and Bayomi M. MHD flow of a micropolar fluid past a stretched permeable surface with heat generation or absorption. Non-linear analysis modeling and control. Vol. 14, no 1, 27-40

[26] Seddek M.A. Finite-element method for effects of chemical reaction, variable viscosity, thermophoresis and heat generation/absorption on a boundary layer hydromagnetic flow with heat and mass transfer over a heat surface. Acta mech. 177,pp. 1-18,2005 
